\title{
A non-parametric estimate of mass 'scoured' in galaxy cores
}

\author{
Philip F. Hopkins ${ }^{1 \star}$ and Lars Hernquist ${ }^{2}$ \\ ${ }^{1}$ Department of Astronomy, University of California Berkeley, Berkeley, CA 94720, USA \\ ${ }^{2}$ Harvard-Smithsonian Center for Astrophysics, 60 Garden Street, Cambridge, MA 02138, USA
}

Accepted 2010 April 24. Received 2010 April 5; in original form 2010 February 11

\begin{abstract}
We present a simple estimate of the mass 'deficits' in cored spheroids, as a function of galaxy mass and radius within the galaxy. Previous attempts to measure such deficits depended on fitting some functional form to the profile at large radii extrapolating inwards; this is sensitive to the assumed functional form and does not allow for variation in nuclear profile shapes. For example, we show that literally interpreting the residuals from a single/cored Sersic function fit as implied 'deficit' can be misleading. Instead, we take advantage of larger data sets to directly construct stellar mass profiles of observed systems and measure the stellar mass enclosed in a series of physical radii $\left(M_{*}(<R)\right)$, for samples of cusp and core spheroids at the same stellar mass. We show that there is a significant (model-independent) bimodality in this distribution of central structure for this sample at small radii. We non-parametrically measure the median offset between core and cusp populations (the 'deficit' $\Delta M_{*}(<R)$ ). We can then construct the scoured mass profile as a function of radius, without reference to any assumed functional form. The mass deficit rises in power-law fashion $\left(\Delta M_{*}(<R) \propto R^{1.3-1.8}\right)$ from a significant but small mass at $R \lesssim 10 \mathrm{pc}$, to asymptote to a maximum $\sim 0.5-2 M_{\mathrm{BH}}$ at $\sim 100 \mathrm{pc}$, where $M_{\mathrm{BH}}$ is the mass of the central, supermassive black hole (BH) hosted by the spheroid. At larger radii there is no statistically significant separation between populations; the upper limit to the cumulative scoured mass at $\sim \mathrm{kpc}$ is $\sim 2-4 M_{\mathrm{BH}}$. This does not depend strongly on stellar mass. The dispersion in $M_{*}(<R)$ appears larger in the core population, possibly reflecting the fact that core scouring increases the scatter in central profile shapes. We measure this broadening effect as a function of radius. The relatively low mass deficits inferred, and characteristic radii, are in good agreement with models of 'scouring' from BH binary systems.
\end{abstract}

Key words: galaxies: active - galaxies: evolution - galaxies: formation - quasars: general cosmology: theory.

\section{INTRODUCTION}

Massive ellipticals appear to exhibit central 'cores' - regions of constant or weakly divergent surface brightness - as opposed to the power-law 'cusps' - more rapidly rising at small radii - common in less massive ellipticals (King 1978; Young et al. 1978; Lauer 1985; Kormendy 1985, 1987; Lauer et al. 1992; Crane et al. 1993; Lauer et al. 1995; Ferrarese et al. 1994, 2006; Côté et al. 2006, 2007). This bimodality in central surface brightness slopes (Gebhardt et al. 1996; Lauer et al. 2007a) participates in a larger, longer-recognized division of the elliptical population: the typical giant, core elliptical rotates slowly, and has 'boxy' isophotal shapes supported by anisotropic velocity dispersions; less massive cusp ellipticals (and S0 galaxies and classical bulges) rotate more rapidly, have more isotropic velocity dispersions and exhibit 'discy' isopho-

^E-mail: phopkins@ astro.berkeley.edu tal shapes (Kormendy \& Illingworth 1982; Davies et al. 1983; Davis et al. 1985; Jedrzejewski, Davies \& Illingworth 1987; Bender 1988; Bender et al. 1989; Bender, Burstein \& Faber 1992; Peletier et al. 1990; Kormendy \& Bender 1996; Faber et al. 1997; Simien \& Prugniel 1997a,b,c; Emsellem et al. 2004, 2007; McDermid et al. 2006; Cappellari et al. 2007).

These differences thus led naturally to the idea, developed in e.g. Faber et al. (1997), Kormendy (1999), Quillen, Bower \& Stritzinger (2000), Rest et al. (2001), Ravindranath et al. (2001), Laine et al. (2003), Lauer et al. (2005), Lauer et al. (2007a), Ferrarese et al. (2006), Côté et al. (2007), Kormendy et al. (2009), that discy, rapidly rotating cusp ellipticals are direct products of gasrich ('wet') mergers, whereas boxy, slowly rotating core ellipticals have been shaped by subsequent dissipationless ('dry') re-mergers of two or more (initially cuspy gas-rich merger remnant) ellipticals. However, numerical experiments and simple phase-space arguments demonstrate that central light profile shapes are, to lowest order, preserved in dissipationless mergers without binary black 
holes (BHs) (e.g. Boylan-Kolchin, Ma \& Quataert 2005; Hopkins et al. 2009d). It is therefore generally believed that the connection between the merger history of galaxies and their nuclear profile slope arises because of 'scouring' by a binary BH (for a review, see Gualandris \& Merritt 2007). Begelman, Blandford \& Rees (1980) first pointed out that binary BHs coalescing in a dissipationless galaxy merger could stall (i.e. are no longer efficiently transported to the centre via dynamical friction) at radii $\sim \mathrm{pc}$, larger than the radii at which gravitational radiation can efficiently dissipate energy and merge the binary - the so-called 'last parsec problem'. They noted that significant gas content can provide a continuous source of drag and friction and solve this problem in gas-rich mergers, but that in 'dry' mergers, the binary will remain stalled for some time, and will harden by scattering stars in the nucleus in three-body interactions. This will continue, flattening the nuclear slope, until a sufficient mass in stars $\left(\sim M_{\mathrm{BH}}\right.$, by simple scaling arguments) is ejected to merge the binary.

It is therefore of particular interest to estimate the stellar mass which must be scattered to explain the slopes of cores, as a test of scouring models, a constraint on $\mathrm{BH}-\mathrm{BH}$ merger rates and gravitational in spiral, and as a probe of the galaxy merger history. To date, there have been a few such calculations (see Milosavljević et al. 2002; Ravindranath et al. 2002; Graham 2004; Trujillo et al. 2004; Ferrarese et al. 2006; Lauer et al. 2007a,b; Côté et al. 2007; Kormendy et al. 2009; Kormendy \& Bender 2009); however, these have in some cases reached very different conclusions regarding the absolute mass 'scoured', whether or not this scales with galaxy mass, and even whether or not there is any evidence for a bimodal population at all (as opposed to a smoothly scaling distribution of central profile gradients).

Much of this ambiguity owes to the lack of an a priori physical model for the 'pre-scouring' profile shape. All of the estimates above implicitly assume some specific functional form for the light 'scoured', where the parameters of this functional form are determined from fitting the observed light profile at larger radii and extrapolating inwards. For example, several of the above works fit either a 'cored' Sersic model (Graham et al. 2003) or a Sersic model with some deficit inside a 'cut-off' radius to the large-scale galaxy profile, and then compare the actual light profile with the inwards extrapolation of the single Sersic portion of the fit (implicitly assuming that the 'pre-scouring' light profile followed the continuation of an identical Sersic profile to the outer, post-merger profile).

However, there is no physical motivation for the Sersic model. It is well-known that there is considerable diversity in the central profile shapes within the cusp population - any individual system could deviate widely from this assumption - and moreover the assumption that profiles represent perfect Sersic continuations is, in fact, known to break down for the majority of non-core ellipticals (Faber et al. 1997; Hibbard \& Yun 1999; Rothberg \& Joseph 2004; Trujillo et al. 2002; Ferrarese et al. 2006; Côté et al. 2007; Kormendy et al. 2009; Hopkins et al. 2009b). For example, Hopkins et al. (2008b, 2009c) show that a physically motivated decomposition into a two-component Sersic model more accurately approximates the central structure at $\sim \mathrm{kpc}$ scales in ellipticals. Adopting a slightly different assumed functional form for the profile fit and extrapolation leads to large systematic differences in the 'missing light' properties. In addition, it is well established that core and cusp ellipticals have different profile shapes at radii outside the 'core' (see references above); even if all systems obeyed the assumed specific functional form, the use of the profile at large radii - radii that have no 'information' regarding the scouring process - to anchor the fit could lead to significant differences between the true and inferred pre-scouring profiles (see Milosavljević et al. 2002; Hopkins et al. 2009d).

Moreover, the scouring process is not expected to be identical from one system to the next. The efficiency and effects of scouring, in detail, are functions of the merger history (e.g. number of mergers and distribution of merger mass ratios and orbits; see Quinlan \& Hernquist 1997; Merritt 2006; Sesana, Haardt \& Madau 2007), as well as properties such as the triaxiality and stellar orbital anisotropy distribution as a function of radius (Holley-Bockelmann \& Sigurdsson 2006; Berczik et al. 2006; Perets \& Alexander 2008); numerical simulations suggest that it may not be described by a simple functional form relative to the profile at larger radii (see references above and Perets, Hopman \& Alexander 2007; Zier 2007).

In this paper, we therefore present a non-parametric estimator of the mass affected by $\mathrm{BH}$ scouring, as a function of radius. We construct stellar mass density profiles for a large sample of both cusp and core ellipticals, and use these to determine the stellar mass enclosed within different physical annuli. Considering this distribution in ellipticals of similar mass, we show that - even without any reference to some 'cusp' or 'core' designation or profile fitting - there is a robust bimodality in the distribution of enclosed mass at small radii. We consider non-parametric measures of the median offset between these bimodal peaks; equivalently, the median difference in enclosed mass between cusp and core ellipticals, as a function of radius. This allows us to construct - albeit still with some assumptions - the 'scoured' mass profile, which grows with radius until asymptoting to a maximum scoured mass $\approx 1-2 M_{\mathrm{BH}}$ near $\sim 100 \mathrm{pc}$. We consider this as a function of galaxy mass, and with different methodologies, and show that it is robust. Unlike the above approaches, the methodologies here do not refer to specific profile fits, and account for the diversity of profile shapes within each population. Moreover, this methodology allows us to actually measure the effects of scouring as a function of radius (rather than implicitly assuming them in the fitting); we show there is an approximate power-law behaviour at small radii with an asymptotic effect on the mass profile at larger radii.

We adopt a $\Omega_{\mathrm{M}}=0.3, \Omega_{\Lambda}=0.7, H_{0}=70 \mathrm{~km} \mathrm{~s}^{-1} \mathrm{Mpc}^{-1}$ cosmology, but note that this has little effect on our conclusions.

\section{THE CONCERN: INFERENCE BY FITTED PROFILE SHAPES}

To begin, Fig. 1 illustrates one reason why caution is needed in the approach of fitting galaxy profiles. Begin with a simple toy-model galaxy light profile, a Sersic profile with index $n_{\mathrm{s}}=3$. This is typical of 'cuspy', $\sim L_{*}$ ellipticals. Now imagine that the system undergoes some series of violent events, such that the profile at large radii is altered and an extended envelope is built up, but the profile at small radii is relatively unperturbed. This is a plausible scenario in the absence of core 'scouring': in $N$-body and hydrodynamic simulations of 'dry' mergers, even in major (1:1) mergers, the result to lowest order is that the profile shape in the central regions where the system is very dense tends to be conserved (stars there conserve their specific binding energies; see e.g. Barnes 1988; Hernquist 1992, 1993), while some scattering or 'splashing' of stars to larger radii makes the tail or envelope of the distribution at large radii more extended (see Boylan-Kolchin, Ma \& Quataert 2006; Hopkins et al. 2009d).

The effect is expected even more so if the envelope is built up not by a single very violent event, but by a series of minor mergers and/or mergers where the secondary is much less dense than the primary in which case it is shredded at large radii and contributes directly to 

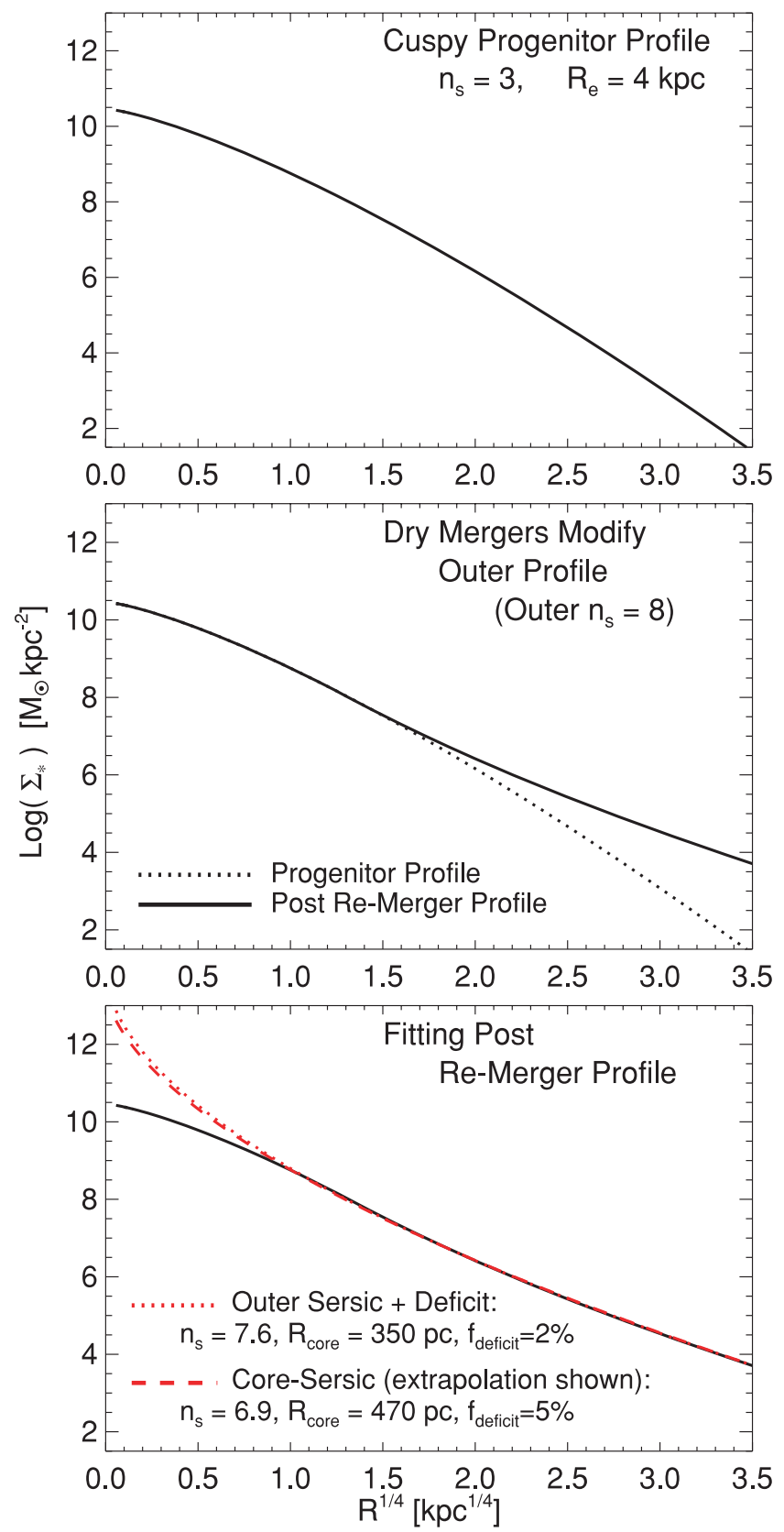

Figure 1. Illustration of caveats in estimating the 'missing' mass in core ellipticals via fitting some assumed functional form to the profile. Top: an idealized profile of a typical cuspy progenitor elliptical: here a perfect $n_{\mathrm{s}}=$ 3 Sersic profile, with the given effective radius. We plot surface stellar mass density versus radius. Middle: one possible way in which subsequent re-mergers and dry mergers will modify the profile, by building up an outer envelope of low-density material (here modelled with an $n_{\mathrm{s}}=8$ profile outside of $R_{e}$ ). There is no change, however, in the central profile shape - in particular, no core 'scouring' here. Bottom: the results of fitting these to assumed functional forms. First, a single Sersic law, fit down from large radii until the variance at a given radius becomes larger than the equivalent of $\Delta \mu=0.1 \mathrm{mag}$. Comparing the inward extrapolation of this to the true profile gives an apparent 'deficit' (mass fraction $f_{\text {deficit }}$ ) inside of this radius $\left(R_{\text {core }}\right)$. Secondly, a core-Sersic profile fit (fitted to the entire profile). We plot the extrapolation of the outer Sersic portion of the fit (the best-fitting full profile with 'core' is indistinguishable from the true profile) with the given outer Sersic index, core break radius and apparent core mass deficit. the envelope, but has a completely negligible effect on the central densities (Gallagher \& Ostriker 1972; Hernquist, Spergel \& Heyl 1993; Naab, Johansson \& Ostriker 2009). Recent evidence from comparison of high-redshift massive spheroids and their descendants today suggests, in fact, that the buildup of such an envelope while central properties are (relatively) weakly affected is probably the dominant evolutionary track for the most massive spheroids (Hopkins et al. 2009a; Bezanson et al. 2009; Hopkins et al. 2010). We mock up such an expansion of the outer envelope by simply modifying the profile such that inside of the original $R_{e}$, it follows exactly the same functional form as before; but at larger radii, it obeys a Sersic law with $n_{\mathrm{s}}=8$ (these choices ensure the profile is smooth through the transition).

Now, fit the relic profile to a new Sersic function. We consider first a single Sersic function fit, following Kormendy et al. (2009) we fit the profile at large radii first, and include more data points to smaller and smaller radii (re-fitting each time) until we reach some radius where the rms deviations about the inward extrapolation of the single Sersic law grow larger than 0.04 dex in $\log \Sigma_{*}$ (roughly equivalent to $\Delta \mu=0.1 \mathrm{mag}$ in surface brightness). Similarly, we can consider the core-Sersic profile

$\Sigma=\Sigma^{\prime}\left[1+\left(\frac{R_{\text {core }}}{R}\right)^{\alpha}\right]^{\gamma / \alpha} \exp \left[-b_{n}\left(\frac{R^{\alpha}+R_{\text {core }}^{\alpha}}{R_{\text {eff }}^{\alpha}}\right)^{1 /(\alpha n)}\right]$

which follows a Sersic function at large radius, with a break to some flatter power-law behaviour at small radii (the fitted $R_{\text {core }}$; Graham et al. 2003), fitted to all radii simultaneously. In either case, the resulting fit defines some outer Sersic profile, and some inner 'core' region, inside of which the true profile falls below the inward extrapolation of the Sersic component.

We show these extrapolations for both cases. They give similar best-fit $n_{\mathrm{s}} \sim 7$ (obviously similar to the input $n_{\mathrm{s}}=8$ profile as $r \rightarrow \infty)$. The inwards extrapolation of each of these fits rises to much larger surface densities than the true profile. Thus, there is an apparent large 'core' or mass deficit inside small radius. Here, the apparent deficit appears at $\sim 400 \mathrm{pc}$, and has a mass or light fraction (defined by simply integrating the light under each curve versus the extrapolated Sersic component) of $\sim 2-5$ per cent.

Of course, the inner regions of our mock profile are, by construction, identical to the progenitor - there has been no scouring of any kind. Assuming that the inwards extrapolation of the fit from large radii represents the progenitor is therefore misleading. Although the example here is purely illustrative (and, by design, extreme), such an effect appears in numerical simulations of dry galaxy-galaxy mergers, for the reasons given above. In Hopkins et al. (2009d), the authors present a large suite of simulations of dry 're-mergers' of simulated elliptical galaxies, those ellipticals (which are then re-merged) themselves the products of gas-rich mergers chosen specifically because of their very close agreement with the observed profile shapes of real observed cusp ellipticals (Hopkins et al. 2009b). After a 1:1 dry re-merger of such systems (with no scouring mechanism included), the authors note that many of the profiles exhibit exactly the sort of changes described above (where the inner profile is approximately conserved, but outer profile 'puffed up'), and can be formally well-fit by a core-Sersic profile. This is despite the fact that there is no core scouring included or resolved in those simulations. Fitting each simulation with such a profile, we show the distribution of mass 'deficits' (defined as above for the core-Sersic fit) and core break radii in Fig. 2. The characteristic deficits of $\sim 0.1-1$ per cent of $M_{\text {gal }}$, and break radii of $\sim 0.01-0.05 R_{e}$, are characteristic of the above issue. 

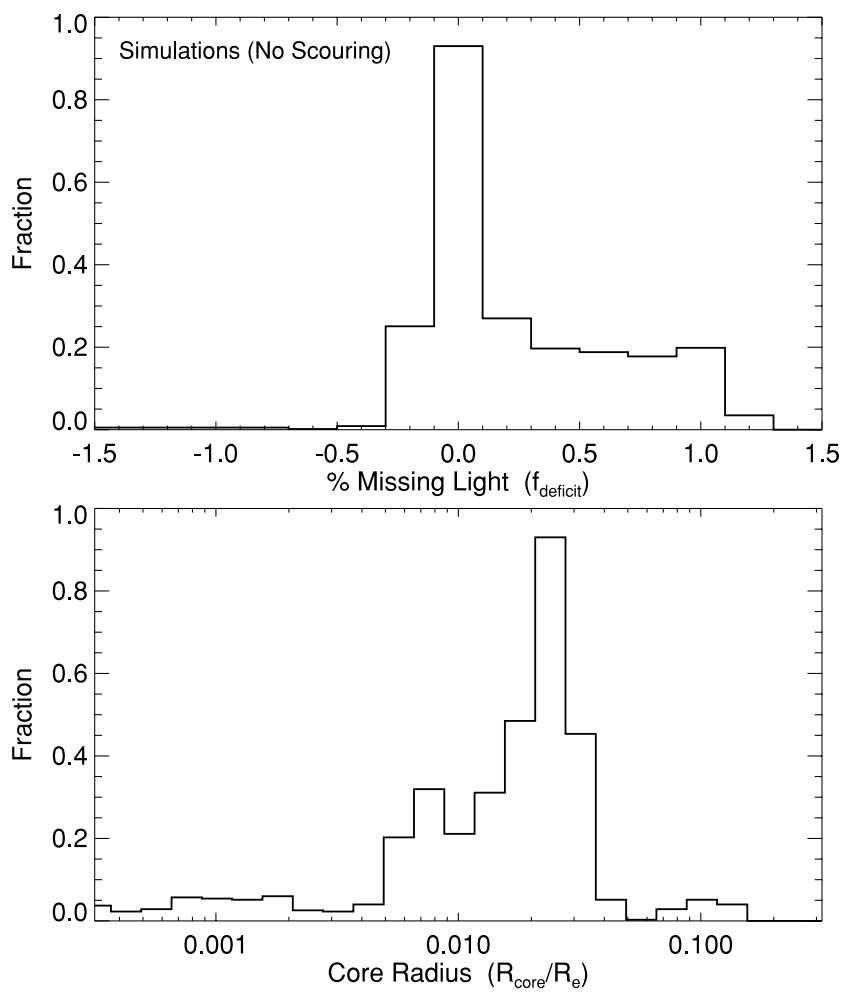

Figure 2. Distribution of 'mass deficit' properties in $\sim 30$ hydrodynamic simulations of major dry merger remnants from Hopkins et al. (2009d). The galaxies are initially in good agreement with profiles of observed cusp ellipticals, and are merged with no scouring mechanism. Post-merger, they are fitted to a core-Sersic profile, with the implied mass deficits and core break radii determined as in Fig. 1. Top: distribution of implied mass deficit percentages. Bottom: distribution of implied core break radii $\left[R_{\text {core }}\right.$ in equation (1), in units of $\left.R_{\text {eff }}\right]$. Owing to the effects in Fig. 1, and simple variation/scatter in the true functional form of galaxy profile shapes, apparent non-trivial mass deficits and large break radii can arise.

The problem arises because of the nature of the Sersic profile, but could generally arise from any extrapolation from larger radii. Fitting such a profile implicitly couples the profile shape at large radii to that at small radii. For a Sersic profile, a more extended envelope at large radii corresponds to a larger index $n_{\mathrm{s}}$. However, a larger $n_{\mathrm{s}}$ also corresponds to a more steeply rising profile at small radii, as is clearly evident in Fig. 1 . The effects on the inferred nuclear 'scoured' mass can be large: an $n_{\mathrm{s}}=2$ profile has just 0.06 per cent ( 3 per cent) of its mass within 1 per cent ( 10 per cent) of $R_{e}$, an $n_{\mathrm{s}}=8$ profile has 2 per cent (14 per cent). Thus, any change in the profile shape at large radii will necessarily change the inferred 'progenitor' profile at small radii. Moreover, observed core ellipticals tend to have significantly higher $n_{\mathrm{s}}$ in their outer regions (more extended envelopes) than lower-mass cusp ellipticals (Trujillo et al. 2002; Ferrarese et al. 2006; Kormendy et al. 2009). This means that, assuming such an inward extrapolation, the assumed 'progenitor' small-scale profile in the cored systems is in fact more steep than that observed in any actual cusp elliptical. For example, the stellar mass profiles of simulated merger remnants in Hopkins et al. (2009b) and Hopkins, Cox \& Hernquist (2008a) are not generically reproduced by a single Sersic function, but rather exhibit multi-component structure indicative of the mixed roles of dissipation and violent relaxation.

\section{METHODOLOGY: AN ESTIMATOR OF THE CORE MASS 'DEFICIT'}

Given these concerns, we desire here to develop a more simplified but hopefully more robust check of mass deficits and core radii.

We begin by considering the surface stellar mass density profiles $\Sigma(R)$ as a function of projected radius $R$ for ellipticals and S0 galaxies with Hubble Space Telescope (HST) imaging of their nuclei (and ground-based profile data at large radii, allowing accurate surface brightness profile measurements from $\lesssim 10 \mathrm{pc}$ to $\sim 50 \mathrm{kpc}$ ). We compile observed surface brightness profiles from Kormendy et al. (2009) and Lauer et al. (2007a); this consists of a total of $\sim 180$ unique local ellipticals. ${ }^{1}$ Inclusion or exclusion of S0 galaxies does not change our conclusions. The HST data allow robust classification of the cusp/core status in each system, discussed therein. We determine stellar masses for each system in Hopkins et al. (2009b), based on the integrated observed optical luminosity in several bands, using the mass-to-light ratio calibrations as a function of colour in Bell et al. (2003) corrected to a Chabrier (2003) initial mass function (IMF). ${ }^{2}$ The isophotally averaged major axis profiles are measured in rest-frame optical; we convert to a stellar mass profile based on the measured total stellar masses and the assumption of a radiusindependent stellar mass-to-light ratio. Most of the objects also have resolved colour gradients - we find that converting to a stellar mass profile using the local colour and a colour-dependent $M_{*} / L$ makes no difference, as the colour gradients are weak. Likewise, conversion to stellar mass profiles using stellar population gradients and comparison of profiles from different instruments and wavebands in these samples are discussed extensively in Hopkins et al. (2008a, 2009b); the differences are much smaller than the variation between individual profiles, and do not affect our conclusions. The Kormendy et al. (2009) sample is a volume-limited survey of the Virgo spheroid population; as such it includes few very massive galaxies $\left(M_{*}>3 \times 10^{11} \mathrm{M}_{\odot}\right)$. The Lauer et al. (2007a) galaxies are chosen to be characteristic of massive ellipticals in the local Universe, including more massive systems up to a couple $10^{12} \mathrm{M}_{\odot}$. At the masses of interest, both are representative of the distribution of spheroid sizes, velocity dispersions and profile shapes in the local Sloan Digital Sky Survey galaxy sample (see e.g. Hyde \& Bernardi 2008).

Fig. 3 illustrates the methodology. First, consider a narrow range in total galaxy stellar mass (here $M_{*}=10^{11.0-11.5} \mathrm{M}_{\odot}$ ), in which there are both cusp and core elliptical populations. Ideally, we would also consider a specific range in $\mathrm{BH}$ mass, but direct $\mathrm{BH}$ mass measurements are available for only a small subset of these objects (too few for the statistics here); as such, we adopt the observed BH-bulge stellar mass relation from Häring \& Rix (2004) to estimate the corresponding $\mathrm{BH}$ masses in these hosts (more on this below). We show a direct comparison of the surface stellar mass density profiles of these systems. We separately denote the cusp and core ellipticals. ${ }^{3}$ We plot results down to the minimum quoted radii from the sources

${ }^{1}$ Note that although the composite ( $H S T+$ ground-based) profiles were used in Lauer et al. (2007a) to estimate effective radii, they were not actually shown in the paper.

${ }^{2}$ Varying the specific bands used to determine stellar masses makes little difference, and changing the IMF will systematically alter the stellar masses of all objects considered, but will not affect our comparisons.

${ }^{3}$ For consistency, we adopt the cusp/core designations in the source samples from Kormendy et al. (2009) and Lauer et al. (2007a), based on their logarithmic nuclear profile slopes at the smallest radii observed. However, other classifications are identical for all but a few objects; adopting those in Ferrarese et al. (2006) or Côté et al. (2007) makes no difference. 

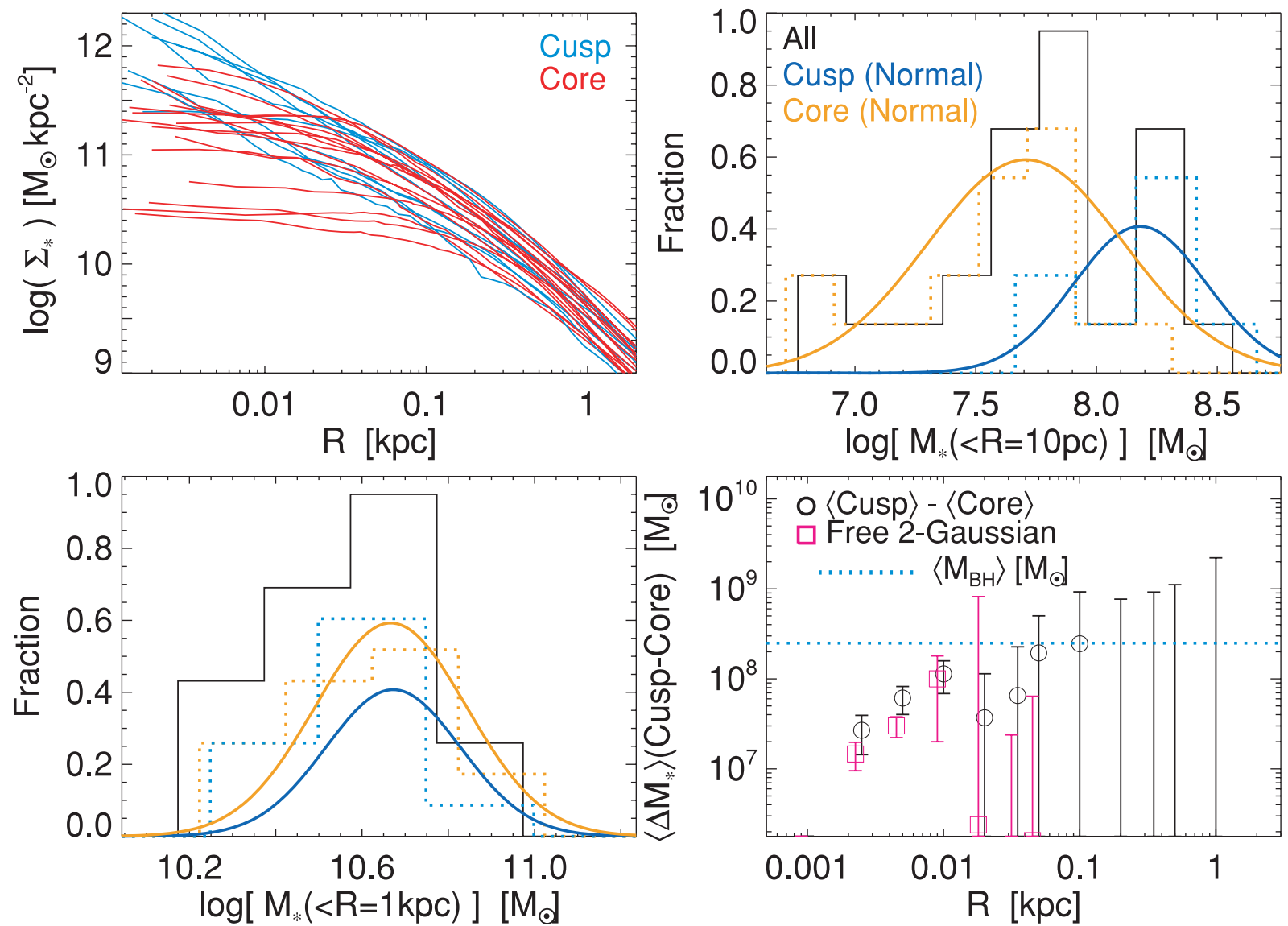

Figure 3. Top left: stellar mass surface density profiles of spheroids in a narrow stellar mass range $M_{*}=10^{11-11.5} \mathrm{M}_{\odot}$. Cusp and core ellipticals are separately noted. Top right: histogram of the enclosed mass $M_{*}(<R)$ inside some radius, determined from the surface density profiles, at $R=10 \mathrm{pc}$. There is a clear bimodality. Gaussian fits to the cusp and core populations are shown separately (histogram of each in dotted lines). Bottom left: same, at $1 \mathrm{kpc}$. Bottom right: difference $\Delta M_{*}$ between the median enclosed mass $M_{*}(<R)$ at each $R$, comparing the cusp and core populations (black circles). Points represent detection of a statistically significant offset in the median $M_{*}(<R)$ between the populations, with the absolute mass noted. Error bars extending below the plotted limits should be considered upper limits; in this case the observations are consistent with no difference. Magenta squares fit the histogram to a double-Gaussian, i.e. assume no prior cusp/core designation (as such the uncertainties are larger); they are plotted only where a double Gaussian is favoured at any significance over a single Gaussian. Compare the average $\mathrm{BH}$ mass in galaxies of this stellar mass range. There is a clear bimodality at small scales, where $\Delta M_{*}(<R)$ grows with $R$ until appearing to asymptote to a maximum $\sim M_{\mathrm{BH}}$ at $R \sim 100 \mathrm{pc}$.

above (the minimum radii at which their seeing-deconvolved profiles are considered reliable) - essentially the HST resolution limit. We stress that we will use these points in all of our analysis below, so some caution is needed in considering how robust the conclusions we quote below are at the smallest radii. In general, resolution limits are such that below $\sim 10 \mathrm{pc}$, the sample size with any resolved points drops dramatically. In the more massive galaxies, the limits are larger; the most massive systems generally are limited to $>30-50 \mathrm{pc}$ resolution. If we simply interpolate the nuclear profiles inwards based on their slope at the minimum resolved radii, our results remain similar down to arbitrarily small radius, but we are specifically attempting to avoid such assumptions about the 'true' profile shape, so in general one should consider the smallest-radii points in each mass bin to be fairly uncertain.

Bearing those caveats in mind, at some fixed radius $R$, we can consider the distribution of stellar mass enclosed within that radius, i.e.

$M_{*}(<R)=\int_{0}^{R} \Sigma\left(R^{\prime}\right) 2 \pi R^{\prime} \mathrm{d} R^{\prime}$.

Note that this is for the projected surface density $\Sigma$. We can also apply an inverse Abel transform to de-convolve the (circularized) projected density profile to recover the three-dimensional density,

$$
\rho(r)=-\frac{1}{\pi} \int_{r}^{\infty} \frac{\mathrm{d} \Sigma}{\mathrm{d} R} \frac{\mathrm{d} R}{\sqrt{R^{2}-r^{2}}}
$$

(Bracewell 1965) and then integrate to determine $M_{*}(<r)$ as the mass within some three-dimensional spherical radius. Because $M_{*}$ is an integral quantity, this makes little difference; in what follows we prefer the use of $M_{*}(<R)$ as it is more numerically stable (the gain in accuracy in de-projecting to a three-dimensional profile is offset by the fact that the Abel transform required in that deprojection is very sensitive to small uncertainties in the measured profile; as such a careful error analysis shows no improvement in the constraints). Fig. 3 shows this for the systems in the given total mass range, at $R=10 \mathrm{pc}$ and $R=1 \mathrm{kpc}$.

We first consider the cusp and core systems separately; Fig. 3 plots the best-fitting Gaussian to each distribution (i.e. Gaussian with the same median and $1 \sigma$ dispersion), and the cumulative distribution (i.e. histogram of all sources, without reference to their cusp/core status). At small radii of $\sim 10 \mathrm{pc}$, there is a clear difference between the distribution of cusp and core enclosed masses - significant at $\sim 5 \sigma$. This is clear directly in the total histogram - there is a striking bimodality in the enclosed mass. 
Comparing a double versus single normal distribution, we find that a distribution with two distinct peaks is preferred at $>4 \sigma$. Other tests yield similar results; for example, the 'dip' test of Hartigan \& Hartigan (1985) favours bimodality at $\sim 3-4 \sigma$ without reference to an assumption of normality in the two-peaked distribution, and can be used to non-parametrically quantify (albeit with larger uncertainties) the peak separation, yielding similar results. This should not be surprising; the statistical significance of the bimodality in central galaxy densities (and their slopes) has been the subject of a number of works (see Ferrarese et al. 1994; Gebhardt et al. 1996; Faber et al. 1997; Lauer et al. 2007a), and is well established in at least this sample of chosen cusp-core galaxies; whether it is smoothed out in strict volume-limited samples (the degree of 'dichotomy' or the uniformity of the transition) is however a question still being debated (see Côté et al. 2007). On the other hand, however, at $1 \mathrm{kpc}$, the distribution is clearly unimodal, and there is no significant difference between cusp and core profiles.

Given this bimodality, we can quantify the difference, on average, between the mass enclosed at small $R$ in the populations. Specifically, we can take the absolute difference between the median $M_{*}(<R)$ for cusp and core ellipticals, with the appropriate uncertainty reflecting both the uncertainty in each median and the weighted uncertainty in the difference. This is non-parametric, and allows us to directly compare cusp and core profiles at a given mass without reference to some model for what the profile 'should be'. Moreover, the large scatter in $M_{*}(<R)$, reflecting the scatter in profile shapes, is properly accounted for - we can robustly quantify the median mass difference in the populations. And it also has the advantage that we can robustly define an error bar on the mass 'deficit'.

Fig. 3 shows the result; on this scale, the two populations are offset by a median $\Delta M_{*}(<R=10 \mathrm{pc})=1.1_{-0.4}^{+0.5} \times 10^{8} \mathrm{M}_{\odot}$. Compare this to the average $\mathrm{BH}$ mass expected and observed in spheroids of this total stellar mass range, $\left\langle M_{\mathrm{BH}}\right\rangle \approx 2.5 \times 10^{8} \mathrm{M}_{\odot}$ (using $M_{\mathrm{BH}} \approx 0.0014 M_{*}$, from Häring \& Rix 2004).

We can be even more conservative, and make no reference to the prior designation of systems as 'cusp' or 'core'. Instead, we simply fit the cumulative distribution of $M_{*}(<R)$ to a double-peaked (double Gaussian) distribution (equivalently, we non-parametrically measure the double-peak separation from other statistical tests as discussed above). In this case, the 'separation' is only well-defined if the double-peaked fit provides a significantly better fit than a single-peaked fit; if a double-Gaussian fit is significantly better (with the attendant 6 degrees of freedom instead of 3), i.e. results in both $\Delta \chi^{2}>1$ and improved $\chi^{2} / \nu$, then we quantify the resulting difference in the median between the two fitted peaks and the formal statistical uncertainty associated. Unfortunately, because the number of objects in any mass bin is small, we find that freeing all the parameters of the fit yields too many degrees of freedom for any but marginally significant results (although the results, averaging over all mass bins, reach $\sim 3 \sigma$ significance and support our conclusions from the prior-constrained comparison of cusp and core populations above).

We therefore show results fixing the relative fraction of the population in each Gaussian to the fraction of cusp or core ellipticals (we have also fixed the relative widths, rather than the fractional population, and find similar results). If the division between cusp and core populations did not reflect some physical bimodality, this measure would give a null result (no signal for statistically significant difference) at all radii; if a large fraction of objects were somehow misclassified, or if an 'intermediate' class exists (i.e. a smooth transition from cusp to core), this method will allow for that (even with our fixing of either the Gaussian widths or relative normalizations), and should still robustly quantify the difference between bimodal peaks in the population. We find similar results to the case above, but with larger uncertainties.

Fig. 3 continues to show these fit results as a function of radius, from $\sim 3 \mathrm{pc}$ to $\sim 10 \mathrm{kpc}$. Again, we recall the cautions stated above regarding the effects of resolution and seeing limits at the smallest radii. At $\gtrsim 100 \mathrm{pc}$, however, the distribution of enclosed masses is clearly unimodal. Specifically, at $\sim 1 \mathrm{kpc}$ (where we show the full distribution) for example, fitting directly the cumulative distribution there is no preference for a doubly peaked underlying distribution. ${ }^{4}$ Even considering the cusp and core populations separately, there is no statistically significant difference between their $M_{*}(<R)$ distributions on this scale. Formally, we find $\Delta M_{*}=-3 \times 10^{7} \mathrm{M}_{\odot}\left(+1.7 \times 10^{9} \mathrm{M}_{\odot}\right)\left(-2.0 \times 10^{8} \mathrm{M}_{\odot}\right)$; in other words, the distributions favour no difference between the two populations, and we can set a 90 per cent upper limit on the core 'deficit' at this radius of $\approx 4 M_{\mathrm{BH}}$.

In Fig. 4, we generalize this to several mass intervals, from $\sim 10^{11}$ to $10^{12} \mathrm{M}_{\odot}$. In each of several mass intervals, we again show the mass profiles of the observed cusp and core systems at small radii. We also show the logarithmic slope of the mass profile, which highlights the flattening in the cusp systems (discussed further below). And we show the median $\Delta M_{*}(<R)$ as a function of radius $R$, determined using the same methodology as in Fig. 3. To put the different mass intervals on the same footing, we show the mass fraction $M_{*}(<R) / M_{*}(<R \rightarrow \infty)$. In these units, the average BH mass implied by the Häring \& Rix (2004) BH-host galaxy mass correlation is a constant $\approx 0.0014$. That is not, of course, the only determination of this relation (compare Magorrian et al. 1998; Marconi \& Hunt 2003), nor is it the unique predictor of BH mass: velocity dispersion (Ferrarese \& Merritt 2000; Gebhardt et al. 2000), host galaxy binding energy or potential well depth (Hopkins et al. 2007a,b; Aller \& Richstone 2007), and profile shape (Graham et al. 2001) are possible alternatives. However, given the relatively limited mass range shown, these predictors do not differ very dramatically over this range. Specifically, we also show the median $M_{\mathrm{BH}} / M_{*}$ expected in each bin using the $M_{\mathrm{BH}}-\sigma$ relation together with the measured $\sigma$ of each galaxy in the subsample. At the highest masses, the median expectation from $M_{\mathrm{BH}}-\sigma$ is a factor of $\sim 2$ lower than that from the $M_{\mathrm{BH}}-M_{\text {bulge }}$ relation, but this is where our error bars become quite large (owing to the dearth of cusp ellipticals at these masses), so our comparison cannot discriminate at any significance whether there is a better correlation between the implied deficits and the $\mathrm{BH}$ host mass implied $\mathrm{BH}$ masses or $\mathrm{BH}-\sigma$ implied masses. It should however be borne in mind that the two estimators will diverge for the most massive systems, near the upper limit of our sample [by $M_{*}=10^{12} \mathrm{M}_{\odot}$ they may differ by a factor of several (Lauer et al. $2007 b$ ) but most of the objects in our most massive bin are concentrated near the low-mass range of the interval].

In each case in Fig. 4, a similar trend is seen. At very small radii $\lesssim 10 \mathrm{pc}$, there is a significant bimodality in the population, but

\footnotetext{
${ }^{4}$ At these radii, caution is needed in adopting the fully freed bimodal fitting approach - if the distribution is slightly non-Gaussian (has some skewness), and one fits a double Gaussian, it is possible to obtain a statistically significant 'offset' that is clearly unrelated to the cusp/core populations. Some constraint like that adopted here is necessary, or more arbitrary functional forms (still unimodal) must be fit in combination, to prevent unphysical results. Given these uncertainties, properly marginalizing over the fitting parameters is particularly important to obtaining valid error estimates.
} 

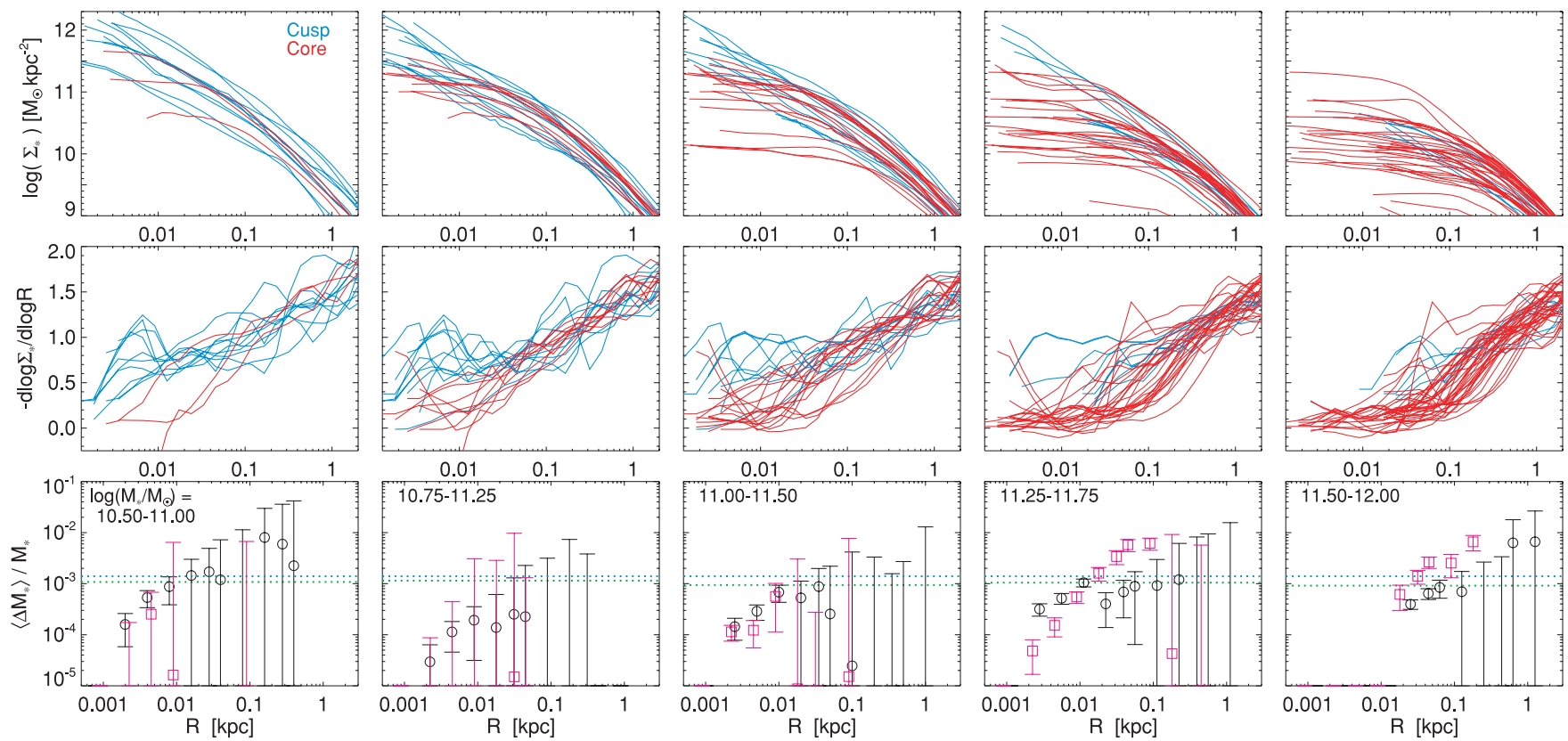

Figure 4. As Fig. 3, for spheroids in different stellar mass intervals. Top: mass profiles. Middle: logarithmic slope of the mass profile, which highlights the degree of 'flattening' in the central regions. Bottom: estimated 'deficit' as in Fig. 3, as a function of radius. To compare systems with different total masses, we plot the fractional mass difference enclosed, i.e. $\Delta M_{*}(<R) / M_{*}$ (normalizing each profile by its stellar mass; because of this, the plotted results for the $\sim 10^{11-11.5} \mathrm{M}_{\odot}$ bin are not exactly identical to those in Fig. 3, though they are very similar). Dotted lines show the expected $\left\langle M_{\mathrm{BH}}\right\rangle$ for the median galaxy stellar mass in the bin and the BH-host mass relation of Häring \& Rix (2004), or the median velocity dispersion $\sigma$ and BH- $\sigma$ relation of Tremaine et al. (2002) (blue and green, respectively). In each mass bin, similar trends are seen: a significant bimodality at small radii with $\Delta M_{*}(<R)$ growing to a maximum detection at $\sim 0.5-2 M_{\mathrm{BH}}$ at $R \sim 100 \mathrm{pc}$; in at least several mass bins, 90 per cent upper limits of $\Delta M_{*}<2-4 M_{\mathrm{BH}}$ are present out to $\sim 0.5 \mathrm{kpc}$.

the amount of mass contained in this radius is small. As such, the absolute mass difference between the cusp/core populations grows to larger and larger radii (very roughly with a power-law index $\Delta M_{*} \propto R^{1.3-1.8}$ ). However, between $\sim 10$ and $100 \mathrm{pc}$, this appears to asymptote to a maximum of $\Delta M_{*} \sim 1-2 M_{\mathrm{BH}}$. Comparing each panel with the appropriate implied $\left\langle M_{\mathrm{BH}}\right\rangle$ from either the $\mathrm{BH}$-host mass or $\mathrm{BH}-\sigma$ relation, $\Delta M_{*}$ appears similar in units of $M_{*}$. At larger radii $R>100 \mathrm{pc}$, there is no statistically significant bimodality remaining. Formally, the difference $\Delta M_{*} \rightarrow 0$ at these radii; if this were strictly the case, it would imply that scoured mass is simply 'kicked' to slightly larger radii (not expelled from the galaxy or moved to very large radii). However, the error bars are still large it is very much possible that the mass is removed entirely, and so the mass difference $\Delta M_{*}$ remains constant at $\sim$ a couple of $M_{\mathrm{BH}}$ at all $R$ (of course, this will become less significant relative to the total enclosed mass at larger $R$ ). But the difference does not appear to grow; the $\sim 90$ per cent upper limit in at least some cases remains at or below $\sim 4 M_{\mathrm{BH}}$ at radii $\sim 0.5-2 \mathrm{kpc}$.

Of course, the comparison of profile shapes makes it immediately clear that the uncertainties at both low and high masses are considerable, owing to the lack of core and cusp spheroids, respectively. Note that the total mass range in Fig. 4 is relatively small, and the individual bins in mass are relatively wide. This is necessary for the statistics needed, and because cusp galaxies become progressively more rare at high masses as cores are more rare at low masses. But together with the limited sample sizes observed at sufficiently high resolution, it means that the difference between the median mass of the cored spheroids in our lowest-mass bin and that of the cusp spheroids in our highest-mass bin is only a factor of $\approx 7$. And recall that the apparent cusps or cores at the mass 'extremes' may also represent unusual growth histories rather than typical progenitors/descendants. The effect of resolution limits is also increasingly evident in the highest-mass interval, where the innermost point of any comparison is at $\approx 20-30$ pc (with the best statistics at $\sim 50-100 \mathrm{pc}$ ). Thus our results at the extremes should be taken with appropriate caution.

Fig. 4 also shows the logarithmic derivative of the mass profile, $-\mathrm{d} \log \Sigma_{*} / \mathrm{d} \log R$, as a function of radius. This highlights the range of behaviour in the central regions of the observed systems. It also reaffirms a number of our results. At large radii, it is clear that it is not just the absolute values of $\Sigma_{*}$ that are similar in all systems in each bin, but also their slopes - this is where we do not see any statistically significant separation. At small radii, however, we see what is expected - the galaxies identified as 'cusp' systems generally maintain slopes in the range $-1 \lesssim \mathrm{d} \log \Sigma_{*} / \mathrm{d} \log R \lesssim-0.5$, down to the resolution limits, whereas those identified as cores fall closer to flat slopes. Of course, there are also intermediate systems in between. Most interesting, the radii at which we see $\Delta M_{*}$ appear to asymptote (or above which do not see a statistically significant difference) appear to roughly correspond to the radii above which the core populations reach slopes steeper than $\mathrm{d} \log \Sigma_{*} / \mathrm{d} \log R \lesssim-0.5$. In the most massive core systems (although it is more ambiguous in the less massive core systems) this is also typically within a factor of $\sim 2$ of the radius where the absolute value of the second derivative of the profile slope is maximized (if it is estimated with some smoothing kernel; we do not show the numerical second derivatives here because they are very noisy).

This is interesting because such a threshold has been proposed and used as an estimator of where 'scouring' action has taken place - if valid, it would allow an assessment of the scouring effects in individual galaxies, without the limitation of large samples required here (see Gebhardt et al. 1996; Lauer et al. 2007b). Of course, we are expressly attempting to avoid making assumptions about the functional form of the pre-scouring profile, so a comprehensive 
comparison of these results to those here is outside the scope of this paper, but the comparison here may lend some motivation to those approaches, if the threshold slope values are taken from comparison similar to that above. More rigorously, we can ask: if we were to adopt some parametric extrapolation of the profile shape on a per-galaxy basis, what would provide the best match to our results? Consider each mass interval above. For each core system, we could assume (with the dangers outlined above inherent in this process) that the pre-scouring profile followed a power law at small radii with approximately constant slope at some $\mathrm{d} \log \Sigma_{*} / \mathrm{d} \log R=\eta_{\text {crit }}$; we simply would estimate the 'pre-scouring' profile by extrapolating the observed profile with $\eta_{\text {crit }}$ at radii below that where the observed profile slope falls below $\eta_{\text {crit }}$. Averaged over the core population in each mass interval, this will give some average $\Delta M_{*}(<R)$ implied - and we can ask what value of $\eta_{\text {crit }}$ minimizes the difference between this estimator and our non-parametric estimator. Performing this exercise for the mass intervals in Fig. 4, we find that in all mass bins, the difference between our estimator and the distribution of deficits recovered by this method is minimized for threshold slopes in the range $-0.5 \lesssim \eta_{\text {crit }} \lesssim-0.8$ (specifically $-0.75,-0.52,-0.81,-0.63,-0.60$, from least to most massive, with respect to the cusp versus core estimator; the error bars are sufficiently large in the two-Gaussian estimator that there is not a strong discriminant in this range). Considering all bins simultaneously, the best-fitting value is $\eta_{\text {crit }} \approx-0.62$ for this sample.

At each radius, we have not just the median $M_{*}(<R)$ for each of the cusp and core populations, but also the dispersion (roughly lognormal) in $M_{*}(<R), \sigma\left[\log \left\{M_{*}(<R)\right\}\right]$. At several radii in the mass bins considered, this dispersion appears to be significantly larger for the core population, as compared to the cusp population. In contrast, there are no cases of statistical significance where the cusp population has larger dispersion. This might be anticipated, given the arguments in Section 1; the scouring process is, in detail, sensitive to quantities such as the merger mass ratio, binary orbital parameters and triaxiality of the host, that will vary even at fixed stellar mass and fixed host mass profile shape. As such, it is plausible that scouring will broaden the distribution of mass profile shapes and correspondingly $M_{*}(<R)$ at small radii, relative to the distribution present in the progenitor population. If both $\sigma\left[\log \left\{M_{*}(<R)\right\}\right]$ for core and cusp populations can be determined, this additional broadening or dispersion term should be given by their difference (subtracted in quadrature). Fig. 5 shows this. Because this requires going to higher-order moments than the median $M_{*}(<R)$, the statistics are correspondingly more limited - we only find a statistically significant result at a couple of radii and masses. But in these cases, the trend is interesting. Reflecting what is seen in the absolute mass differences, the 'additional' dispersion term appears to grow at small radii (although it should be stressed that the dispersion in both populations grows at small radius; see Hopkins et al. 2009e). At $\sim 1-10 \mathrm{pc}$, scattering processes may lead to fractional dispersion in $\Delta M_{*}(<R)$ at the $\sim 0.5$ dex level. In absolute terms, this amounts to dispersion, at these radii, of $\sim M_{\mathrm{BH}}$ in the absolute mass differences. At larger radii, the fractional dispersion falls (as it must, if the absolute mass scattered does not significantly exceed $\left.\sim M_{\mathrm{BH}}\right)$.

\section{DISCUSSION}

We illustrate important systematic uncertainties in the mechanism of inferring the 'mass deficits' at the centres of cored spheroids via fitting of prior assumed functional forms to the mass profile (the 'core' in the 'core-Sersic' profile), and show that these can in princi-

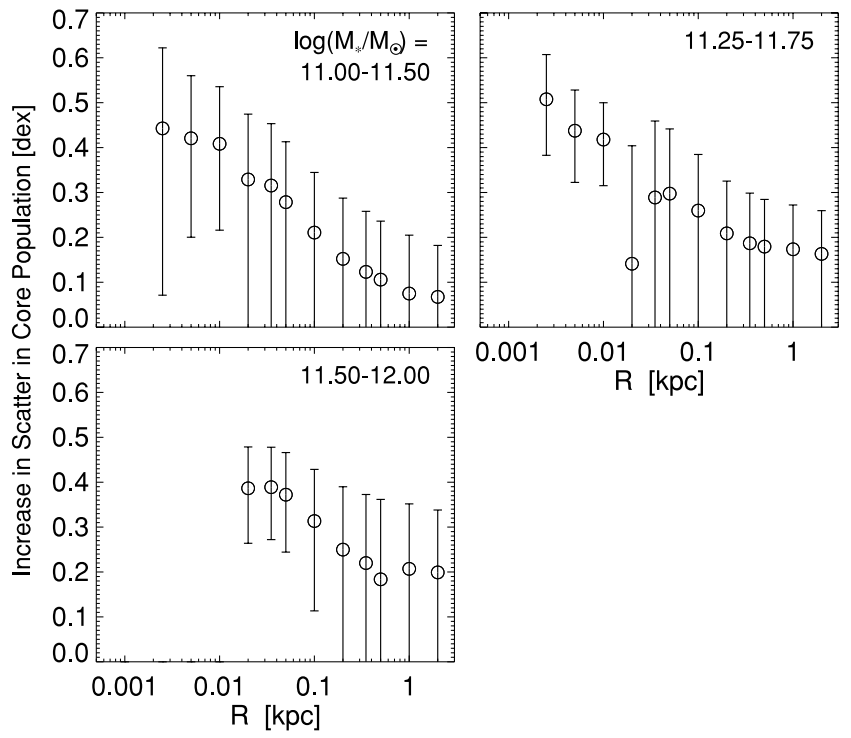

Figure 5. Difference in the logarithmic dispersion in $M_{*}(<R)$ in cusp and core populations $\left(\sigma\left[\log \left\{M_{*}(<R)\right\}\right]\right.$, for each population, subtracted in quadrature). Where this is $>0$, it implies a significant broadening of the dispersion in $M_{*}(<R)$ by the plotted dispersion. Style is as in Fig. 4; we exclude the two low-mass bins as they show no statistically significant detection at any radius. We find no statistically significant cases where the dispersion in core systems is more narrow than that in cusp systems. The observations tentatively show evidence for non-trivial variation in scouring processes at small radii.

ple lead to large artificial mass deficits and large apparent core radii. Specifically, we show that the assumption that the 'un-scoured' progenitor profile matches the inwards extrapolation of a single Sersic fit to the galaxy profile at larger radii can be problematic, and is often misleading when applied to simulated galaxy profiles.

To avoid these uncertainties, we therefore define a simple, nonparametric measure to compare the stellar mass difference in the centre of cored versus cusp spheroids. Essentially, this amounts to a comparison of the enclosed mass profiles $M_{*}(<R)$, quantifying the significance of bimodality in this distribution and, given that, the separation between bimodal peaks. This does not rely on any prior assumption regarding the functional form of the 'true' prescouring profile (only the concept that core ellipticals descend from cusp ellipticals similar to those present today); nor does the inferred mass deficit depend on the profile behaviour at larger radii. It also allows us to account for the non-trivial dispersion in central profile shapes within the cusp and core populations.

We stress, however, that we are still making a very important assumption: namely that the cusp ellipticals present today, in a given stellar mass bin, are representative of the true progenitors of the core ellipticals present today at similar stellar mass. This is not necessarily the case! The progenitor galaxies had to form earlier than their descendants (and the stellar populations of the core population appear older, on average, than those of the cusp population). So it is not hard to imagine, for example, that the core progenitors formed from most gas-rich events at high redshift, and/or that they were more concentrated and compact, as observed spheroids at these redshifts (e.g. Williams et al. 2010, and references therein). Moreover, some of the cusp population at high masses may represent cored galaxies that had their cusps 'rebuilt' by new inflows from subsequent gas-rich mergers and/or cooling flows. Indeed, structural evolution in the outer parts of these galaxies, 
and subsequent mergers, is expected in most theoretical models (Hopkins et al. 2010; Naab et al. 2009). It does appear, however, that the central portions from $\sim 1$ to $5 \mathrm{kpc}$ of even the most compact $z>2$ spheroids are similar in their stellar mass densities and profile shapes to local massive spheroids (Hopkins et al. 2009a), so our implicit assumption may be justified. Nevertheless, an ideal comparison would contrast present-day cored spheroids with higher-redshift candidate progenitors, chosen on the basis of a combination of mass and clustering or other constraints such that they are likely to evolve into the present-day core population. Unfortunately, there are no present observations that can resolve the cusp/core structure of these high-redshift systems. Therefore, we are limited to the present-day cusp population as our best-guess reference set, and should be careful to interpret our results in this context.

With that caveat in mind, we show that there is a clear trend in the comparison of present-day nuclear profiles: at small radii, the distribution of enclosed mass in chosen cusp-core samples is bimodal at high significance. The mean mass difference - the average 'scoured' mass, in the scouring interpretation - between the two bimodal peaks is low at small radii ( $\ll M_{\mathrm{BH}}$ at $\left.R \sim 3-10 \mathrm{pc}\right)$, reflecting the fact that there is simply little absolute mass in such a small enclosed volume. The apparent 'scoured' mass then grows with radius (roughly as $\propto R^{1.3-1.8}$ ) until $\sim 10-100 \mathrm{pc}$, at which point it asymptotes to a constant mass fraction $\approx 1-2 M_{\mathrm{BH}}\left(\sim 10^{-3}\right.$ to $\left.10^{-2.5} M_{*}\right)$. The fact that we see this 'turnover' or asymptotic behaviour suggests that we are actually resolving the total scoured mass, and radii affected. At larger radii $\gg 100 \mathrm{pc}$, there is no statistically significant signal for bimodality, and the $\sim 90$ per cent upper limits on the scoured mass remain consistent with the same asymptotic mass difference. Neither the scoured mass fraction nor characteristic radii appear to scale strongly with galaxy mass; however, our dynamic range is limited to the mass range over which both cusp and core populations exist in significant numbers $\left(\sim 10^{10.5-11.5} \mathrm{M}_{\odot}\right)$. The characteristic scouring radius, for example, may well scale with the $\mathrm{BH}$ radius of influence $R_{\mathrm{BH}} \sim G M_{\mathrm{BH}} \sigma^{-2} \propto M_{*}^{0.5}$, and in Figs 45 , there is a tentative hint of this (it appears that the characteristic radius increases with mass), but the difference is not statistically significant over this dynamic range with the present statistics.

These results are consistent with the expectation from $N$-body experiments of the effects of a binary $\mathrm{BH}$ on the central stellar mass distribution. Such experiments typically find that the stellar mass scattered before the binary $\mathrm{BH}$ merges is $\sim 0.5-1.5 M_{\mathrm{BH}}$ (with dependence in that range on the central structural properties of the galaxy and structure of the inspiralling mass; see Merritt 2006; Perets \& Alexander 2008, and references therein). The characteristic radii are also consistent; such simulations typically find that although stars from larger radii can contribute significantly to $\mathrm{BH}$ coalescence if they are on long radial box orbits in a triaxial potential (Quinlan \& Hernquist 1997) or if there is a strong perturber (Perets et al. 2007), a significant impact on the mass profile is restricted to radii where the initial $M_{*}(<R) \lesssim M_{\mathrm{BH}}$; for a Hernquist (1990) or $r^{1 / 4}$ bulge, this is approximately below $R / R_{\text {eff }} \approx 0.5 \sqrt{M_{\mathrm{BH}} / M_{*}}$ or $\approx 100$ pc for an $\sim L_{*}$ bulge.

Comparing this to studies where the mass 'deficit' $\Delta M_{*}$ is determined from profile fitting, we find a smaller $\Delta M_{*}$ (and smaller scoured radii) than some. Ferrarese et al. (2006) and Kormendy \& Bender (2009) estimate $\Delta M_{*}$ in a sub-set of the galaxies here, by comparing the actual light profile to the inward extrapolation of Sersic functions fitted to larger radii, they find $\Delta M_{*} \sim 2-4 M_{\mathrm{BH}}$ and $\sim(10-20) M_{\mathrm{BH}}$, respectively. Graham (2004) uses a similar methodology and estimates $\Delta M_{*} \sim 1-4 M_{\mathrm{BH}}$, but from a smaller sample. However, we show in Section 2 that these differences can arise as a systematic effect of the assumed profile shape. Fundamentally, at present, any such choice is somewhat arbitrary. By assuming a specific functional form, the fits implicitly couple the assumed 'progenitor' profile at small radii to the profile at large radii; for the Sersic fit, a galaxy with a more extended envelope (higher $n_{\mathrm{s}}$ ) will necessarily imply a more steep nuclear Sersic profile extrapolation. Thus, for the same true nuclear profile, the inferred mass deficit and core radius increase. In fact, in both samples, the observed core ellipticals have more extended envelopes and higher $n_{\mathrm{s}}$ than essentially any of the cusp elliptical population - thus the inward extrapolation of their Sersic profiles is not necessarily identical to the typical nuclear shape in cusps.

Lauer et al. (2007b) and Milosavljević et al. (2002) consider instead the absolute mass enclosed within the radius where the profile steepens beyond a specified logarithmic slope $\left(\mathrm{d} \ln I_{v} / \mathrm{d} \ln r=\right.$ -0.5 ) or relative to the inwards extrapolation of a power-law (as opposed to Sersic) fit. They find $\Delta M_{*} \approx 2.5 M_{\mathrm{BH}}$ and $\sim 0.5-10 M_{\mathrm{BH}}$, respectively. Both cases, however, still require some implicit a priori assumption of a 'correct' functional form for the progenitor light profile at small radii (or the characteristic shape of 'scoured' regions). Moreover, in this approach, the mass measured is really the post-scouring mass inside this radius, not necessarily the mass removed (a non-trivial difference at the factor of $\sim 2-4$ level).

In addition, the nature of the relatively flat profile at small radii means that whenever some functional form for the progenitor light profile is assumed, most of the 'missing light' will come from the radii very near where the core breaks downwards relative to the 'noncore' profile. As such, the inferred mass deficits are quite sensitive to the exact parametrization of the extrapolated profile near this radius (or, for a fixed slope cut-off, to the exact slope cut chosen). For the core-Sersic law, for example, there is a degeneracy between the fitted core break steepness parameter and the inner profile slope; small variations and common choices in fixing or freeing one of those parameters systematically affect the inferred mass deficits and radii. Our approach avoids this degeneracy; however, it remains difficult to statistically determine whether scouring has taken place at large radii where the enclosed mass is much larger than $M_{\mathrm{BH}}$.

That being said, we do find that the median trend of mass deficit with radius, and absolute value inferred as a function of mass, can be approximately reproduced with the simple assumption that the 'progenitor' profile represents a power-law continuation of the nuclear slope with some minimum $\mathrm{d} \ln I_{v} / \mathrm{d} \ln r \approx-0.6$, at radii where the slope flattens below this value. This is, of course, an assumption of the sort we are trying to avoid, but it does allow for estimation of the mass 'deficit' on an individual galaxy basis, and our comparison here provides at least some justification for such a methodology. This also explains why our results are similar to those in Lauer et al. (2007b) and Milosavljević et al. (2002). It would be of considerable physical interest to see whether or not there is any physical motivation for such a 'characteristic' slope - for example, it has been suggested that self-regulating inflow processes might set a characteristic slope around this value, in inflows driven by gravitational instabilities (Hopkins \& Quataert 2009, 2010).

We stress that we agree with essentially all of the qualitative conclusions of the works discussed above. However, the resulting quantitative distinctions and possible systematics are important as tests of the theoretical models for core creation via BH binary mergers; very large $\Delta M_{*}$ and/or apparent core radii $\sim \mathrm{kpc}$ are actually quite difficult for models of core scouring to explain. Our more conservative study here therefore presents a natural and important complement to these works. We robustly set limits on the median scouring mass as a function of galaxy mass and radius without 
reference to any specific functional form for profile fitting. Moreover, to the extent that these measurements can be improved, they hold the potential to constrain not just the total mass that has been affected by scouring, but actually the scouring 'kernel' - i.e. the magnitude of the effect as a function of radius, which can break a number of degeneracies between theoretical models (see the discussion in Perets \& Alexander 2008).

Clearly, the very simple methodology presented here has significant limitations. We find even with a sample of $\sim 200$ well-observed spheroids that the statistical significance of our results is limited by the number of observed systems, because we can only consider systems in a narrow stellar mass range, and must in that range have a significant population of both cusp and core systems. As such, improving these constraints will require extending these samples with more observations of rare low-mass core or high-mass cusp ellipticals. Increasing the number of systems will also extend the radial range over which constraints can be applied; at some radius $R$ with an average enclosed mass $M_{*}(<R)$, the expected fractional offset between populations will be $\sim M_{\mathrm{BH}} / M_{*}(<R)-$ this must be $\gtrsim 1 / \sqrt{N}$ for a significant detection of the residual effects of 'scouring' at these radii. In particular, determining whether or not the scoured mass is really ejected to large radii (or unbound) or simply kicked to moderate $\sim 0.1-1 \mathrm{kpc}$ radii will require extended samples along these lines. With such observations, study of the 'scouring profile' $\left(\Delta M_{*}(<R)\right)$ can be used to determine the differential contribution to scouring/BH binary coalescence from stars at different radii. This will allow direct observational probes of the loss cone, in particular of which stellar radii and orbits contribute.

The methodology here also only constrains the median mass difference between populations - it cannot be used on an object-byobject basis. But with significantly larger samples, such that not just the median but the scatter and shape of each distribution $M_{*}(<R)$ can be determined, then the variation in scouring effects can be constrained (at least in a statistical sense).

Independent tests are also important. For example, scouring is expected to preferentially eliminate stars on radial orbits and leave a bias for tangential orbits (Quinlan \& Hernquist 1997). Gebhardt et al. (2003) see tentative evidence for this in a limited sample of ellipticals; the major-axis radii within which the effect appears are generally $\sim 0.5-3 R_{\mathrm{BH}}$, similar to our estimates here. Ultimately, a combination of these independent constraints and the non-parametric constraints presented here can be used to determine whether or not, and if so which, parametric approach(es) are most faithful to the true scoured mass, and motivate their use on an object-by-object basis.

\section{ACKNOWLEDGMENTS}

We thank Hagai Perets for insightful comments in the development of this manuscript. We also thank John Kormendy, Tod Lauer, Eliot Quataert and Norm Murray for helpful discussions at various stages in this and related work. Support for PFH was provided by the Miller Institute for Basic Research in Science, University of California Berkeley. EQ is supported in part by NASA grant NNG06GI68G and the David and Lucile Packard Foundation.

\section{REFERENCES}

Aller M. C., Richstone D. O., 2007, ApJ, 665, 120

Barnes J. E., 1988, ApJ, 331, 699

Begelman M. C., Blandford R. D., Rees M. J., 1980, Nat, 287, 307

Bell E. F., McIntosh D. H., Katz N., Weinberg M. D., 2003, ApJS, 149, 289
Bender R., 1988, A\&A, 193, L7

Bender R., Surma P., Doebereiner S., Moellenhoff C., Madejsky R., 1989, A\&A, 217, 35

Bender R., Burstein D., Faber S. M., 1992, ApJ, 399, 462

Berczik P., Merritt D., Spurzem R., Bischof H.-P., 2006, ApJ, 642, L21

Bezanson R., van Dokkum P. G., Tal T., Marchesini D., Kriek M., Franx M., Coppi P., 2009, ApJ, 697, 1290

Boylan-Kolchin M., Ma C.-P., Quataert E., 2005, MNRAS, 362, 184

Boylan-Kolchin M., Ma C.-P., Quataert E., 2006, MNRAS, 369, 1081

Bracewell R., 1965, The Fourier Transform and its Applications. McGrawHill Electrical and Electronic Engineering Series. McGraw-Hill, New York

Cappellari M. et al., 2007, MNRAS, 379, 418

Chabrier G., 2003, PASP, 115, 763

Côté P. et al., 2006, ApJS, 165, 57

Côté P. et al., 2007, ApJ, 671, 1456

Crane P. et al., 1993, AJ, 106, 1371

Davies R. L., Efstathiou G., Fall S. M., Illingworth G., Schechter P. L., 1983, ApJ, 266, 41

Davis L. E., Cawson M., Davies R. L., Illingworth G., 1985, AJ, 90, 169

Emsellem E. et al., 2004, MNRAS, 352, 721

Emsellem E. et al., 2007, MNRAS, 379, 401

Faber S. M. et al., 1997, AJ, 114, 1771

Ferrarese L., Merritt D., 2000, ApJ, 539, L9

Ferrarese L., van den Bosch F. C., Ford H. C., Jaffe W., O'Connell R. W., 1994, AJ, 108, 1598

Ferrarese L. et al., 2006, ApJS, 164, 334

Gallagher J. S. III, Ostriker J. P., 1972, AJ, 77, 288

Gebhardt K. et al., 1996, AJ, 112, 105

Gebhardt K. et al., 2000, ApJ, 539, L13

Gebhardt K. et al., 2003, ApJ, 583, 92

Graham A. W., 2004, ApJ, 613, L33

Graham A. W., Erwin P., Caon N., Trujillo I., 2001, ApJ, 563, L11

Graham A. W., Erwin P., Trujillo I., Asensio Ramos A., 2003, AJ, 125, 2951

Gualandris A., Merritt D., 2007, in Livio M., Koekemoer A. M., eds, Black Holes. STScI Spring Symposium (arXiv:0708.3083)

Häring N., Rix H.-W., 2004, ApJ, 604, L89

Hartigan J. A., Hartigan P. M., 1985, Ann. Stat., 13, 70

Hernquist L., 1990, ApJ, 356, 359

Hernquist L., 1992, ApJ, 400, 460

Hernquist L., 1993, ApJ, 409, 548

Hernquist L., Spergel D. N., Heyl J. S., 1993, ApJ, 416, 415

Hibbard J. E., Yun M. S., 1999, ApJ, 522, L93

Holley-Bockelmann K., Sigurdsson S., 2006, preprint (arXiv:astro$\mathrm{ph} / 0601520$ )

Hopkins P. F., Quataert E., 2009, MNRAS, in press (arXiv:0912.3257)

Hopkins P. F., Quataert E., 2010, MNRAS, doi:10.1111/j.17453933.2010.00855.x

Hopkins P. F., Hernquist L., Cox T. J., Robertson B., Krause E., 2007a, ApJ, 669,45

Hopkins P. F., Hernquist L., Cox T. J., Robertson B., Krause E., 2007b, ApJ, 669,67

Hopkins P. F., Cox T. J., Hernquist L., 2008a, ApJ, 689, 17

Hopkins P. F., Hernquist L., Cox T. J., Dutta S. N., Rothberg B., 2008b, ApJ, 679, 156

Hopkins P. F., Bundy K., Murray N., Quataert E., Lauer T. R., Ma C.-P., 2009a, MNRAS, 398, 898

Hopkins P. F., Cox T. J., Dutta S. N., Hernquist L., Kormendy J., Lauer T. R., 2009b, ApJS, 181, 135

Hopkins P. F., Hernquist L., Cox T. J., Kereš D., Wuyts S., 2009c, ApJ, 691, 1424

Hopkins P. F., Lauer T. R., Cox T. J., Hernquist L., Kormendy J., 2009d, ApJS, 181, 486

Hopkins P. F., Murray N., Thompson T. A., 2009e, MNRAS, 398, 303

Hopkins P. F., Bundy K., Hernquist L., Wuyts S., Cox T. J., 2010, MNRAS, 401, 1099

Hyde J. B., Bernardi M., 2008, MNRAS, in press (arXiv:0810.4924)

Jedrzejewski R. I., Davies R. L., Illingworth G. D., 1987, AJ, 94, 1508 
King I. R., 1978, ApJ, 222, 1

Kormendy J., 1985, ApJ, 292, L9

Kormendy J., 1987, in de Zeeuw P. T., ed., IAU Symp. Vol. 127, Structure and Dynamics of Elliptical Galaxies. D. Reidel Publishing C., Dordrecht, p. 17

Kormendy J., 1999, in Merritt D. R., Valluri M., Sellwood J. A., eds, ASP Conf. Ser. Vol. 182, Galaxy Dynamics - A Rutgers Symposium, Astron. Soc. Pac., San Francisco, p. 124

Kormendy J., Bender R., 1996, ApJ, 464, L119

Kormendy J., Bender R., 2009, ApJ, 691, L142

Kormendy J., Illingworth G., 1982, ApJ, 256, 460

Kormendy J., Fisher D. B., Cornell M. E., Bender R., 2009, ApJS, 182, 216

Laine S., van der Marel R. P., Lauer T. R., Postman M., O’Dea C. P., Owen F. N., 2003, AJ, 125, 478

Lauer T. R., 1985, ApJ, 292, 104

Lauer T. R. et al., 1992, AJ, 103, 703

Lauer T. R. et al., 1995, AJ, 110, 2622

Lauer T. R. et al., 2005, AJ, 129, 2138

Lauer T. R. et al., 2007a, ApJ, 664, 226

Lauer T. R. et al., 2007b, ApJ, 662, 808

McDermid R. M. et al., 2006, MNRAS, 373, 906

Magorrian J. et al., 1998, AJ, 115, 2285

Marconi A., Hunt L. K., 2003, ApJ, 589, L21

Merritt D., 2006, ApJ, 648, 976

Milosavljević M., Merritt D., Rest A., van den Bosch F. C., 2002, MNRAS, 331, L51

Naab T., Johansson P. H., Ostriker J. P., 2009, ApJ, 699, L178
Peletier R. F., Davies R. L., Illingworth G. D., Davis L. E., Cawson M., 1990, AJ, 100, 1091

Perets H. B., Alexander T., 2008, ApJ, 677, 146

Perets H. B., Hopman C., Alexander T., 2007, ApJ, 656, 709

Quillen A. C., Bower G. A., Stritzinger M., 2000, ApJS, 128, 85

Quinlan G. D., Hernquist L., 1997, New Astron., 2, 533

Ravindranath S., Ho L. C., Peng C. Y., Filippenko A. V., Sargent W. L. W., 2001, AJ, 122, 653

Ravindranath S., Ho L. C., Filippenko A. V., 2002, ApJ, 566, 801

Rest A., van den Bosch F. C., Jaffe W., Tran H., Tsvetanov Z., Ford H. C., Davies J., Schafer J., 2001, AJ, 121, 2431

Rothberg B., Joseph R. D., 2004, AJ, 128, 2098

Sesana A., Haardt F., Madau P., 2007, ApJ, 660, 546

Simien F., Prugniel P., 1997a, A\&AS, 122, 521

Simien F., Prugniel P., 1997b, A\&AS, 126, 15

Simien F., Prugniel P., 1997c, A\&AS, 126, 519

Tremaine S. et al., 2002, ApJ, 574, 740

Trujillo I., Asensio Ramos A., Rubiño-Martín J. A., Graham A. W., Aguerri J. A. L., Cepa J., Gutiérrez C. M., 2002, MNRAS, 333, 510

Trujillo I., Erwin P., Asensio Ramos A., Graham A. W., 2004, AJ, 127, 1917 Williams R. J., Quadri R. F., Franx M., van Dokkum P., Toft S., Kriek M., Labbé I., 2010, ApJ, 713, 738

Young P. J., Westphal J. A., Kristian J., Wilson C. P., Landauer F. P., 1978, ApJ, 221, 721

Zier C., 2007, MNRAS, 378, 1309

This paper has been typeset from a $\mathrm{T}_{\mathrm{E}} \mathrm{X} / \mathrm{L} \mathrm{T}_{\mathrm{E}} \mathrm{X}$ file prepared by the author. 